\title{
Small-World Optimization Algorithm and Its Application in a Sequencing Problem of Painted Body Storage in a Car Company
}

\author{
Tian Zhipeng, ${ }^{1}$ Shao Xinyu, ${ }^{1}$ Zhu Haiping, ${ }^{1}$ Yin Hui, ${ }^{1}$ and He Fei ${ }^{2}$ \\ ${ }^{1}$ State Key Laboratory of Digital Manufacturing Equipment \& Technology, Huazhong University of Science \& Technology, \\ Wuhan 430074, China \\ ${ }^{2}$ Nanjing University of Science and Technology, Nanjing, Jiangsu 210000, China
}

Correspondence should be addressed to Zhu Haiping; haipzhu@hust.edu.cn

Received 6 June 2014; Accepted 13 October 2014

Academic Editor: Kang Li

Copyright (C) 2015 Tian Zhipeng et al. This is an open access article distributed under the Creative Commons Attribution License, which permits unrestricted use, distribution, and reproduction in any medium, provided the original work is properly cited.

\begin{abstract}
In the car company, the painted body storage (PBS) is set up between the paint shop and the assembly shop. It stores the vehicles in production and reorders the vehicles sequence. To improve production efficiency of assembly shop, a mathematical model is developed aiming at minimizing the consumption rate of options and the total overtime and idle time. As the PBS sequencing process contains upstream sequence inbound and downstream sequence outbound, this paper proposes an algorithm with two phases. In the first phase, the discrete small-world optimization algorithm (DSWOA) is applied to schedule the inbound sequence by employing the short-range nodes and the long-range nodes in order to realize the global searching. In the second phase, the heuristic algorithm is applied to schedule the outbound sequencing. The proposed model and algorithm are applied in an automobile enterprise. The results indicate that the two-phase algorithm is suitable for the PBS sequencing problem and the DSWOA has a better searching performance than GA in this problem. The sensitivity of model parameters is analyzed as well.
\end{abstract}

\section{Introduction}

Motivated by the diversified demands from customers, the make-to-order, multiple variety, and small batch production model gains a wide application in the automotive industry and brings the mixed-model assembly lines. In an automotive assembly plant, the production lines mainly consist of four kinds of shops: the press shop, the welding shop, the paint shop, and the assembly shop. Since the processing time of different types of vehicles is different, the storage is set up between the workshops to guarantee the safety lead time of the next shop [1].

Besides storing, the storage reorders the production sequence also [2]. A large number of studies have been done on sequencing problem with considering the storage. These studies mainly focus on two aspects: the modeling method of the storage and the optimization algorithms to the model.

Jayaraman et al. [3] simulated and designed the capacity of the storage according to the requirements of sequencing capacity and proved the effectiveness of the method with a given case. Kavusturucu and Gupta [4] researched the manufacturing system of limited storage serial. They computed the production capacity of the system using the methods of decomposition, isolation, and expansion and then compared their results with simulation results. Aksoy and Gupta [5] studied the manufacturing cell with limited storage and unreliable machine. Based on decomposition principle and extension method, a scheme of the approximate optimal storage allocation was proposed. Bulgak [6] studied the interstage storage of open loop step assembly line and obtained the optimal solution on the basis of metamodeling and genetic algorithm.

With the establishment of the storage, more scholars have focused on the sequencing function of storage. Muhl et al. [7] resolved the storage sequencing problems through a heuristic algorithm and then provided some suggestions for the problems. Spieckermann et al. [8] researched the linear storage sequencing between the body shop and the paint shop to minimize the times of color switching in the paint shop. Moon et al. [9] put forward a storage/retrieval algorithm to 
solve the linear storage sequencing between the paint shop and assembly shop and verified the algorithm with simulation modes.

Storage sequencing problems can be classified into the discrete problems. In discrete optimization fields, there are many mature algorithms to solve sequencing problem, and we usually use heuristic algorithms such as ant colony algorithm and genetic algorithm. When solving the discrete sequencing problem, the ant colony algorithm may be convergence to local optimum and the genetic algorithm has low solving efficiency as producing a lot of redundancy iteration by using insufficient feedback information. Therefore, we need an algorithm which has a strong capability of global searching and fast convergence speed. Discrete small-world optimization just meets our requirements.

The earliest research on the small-world originated in 1929 from a Hungarian writer F. Karinthy, who conjectured that in this world any two persons could be related by a chain composed of five contacts [10]. In 1967, Milgram confirmed this conjecture by the famous letters delivery experiments and proposed the theory of Six Degrees of Separation [11]. Watts and Strogatz first established the small-world network model, which is based on a ring lattice and called W-S model $[12,13]$. In W-S model, the edges of the network are divided into "local" and "long-range" contacts.

As the small-world network theory system is gradually maturing, the small-world networks have begun to be used in the optimization field. Walsh [14] studied the application of the small-world network on the problem of graph theory search. He improved the small-world networks to adapt the graph theory search. Chen et al. [15] pointed out that different values of the spread probability parameters will affect the quick searching feature of small-world network. A smallworld topology structure was applied in PSO by Cui et al. [16] to observe the behavior of swarm. This method has made PSO more efficient than before. Li et al. [17] proposed a decimalcoding small-world optimization algorithm (DSWOA) based on Kleinberg's model and demonstrated its stability and fast convertible rate for high-dimensional optimization problems. Li et al. [18] developed a small-world hierarchical tree model, which took advantage of characteristics of social network, aiming at solving the continuous optimization problem. Inspired by the intelligent experiments of Milgram's letters delivery and its application in continuous optimization problem, we propose a fast search algorithm based on smallworld effect for discrete problems called discrete small-world optimization algorithm (DSWOA).

This paper focuses on a mixed-model production line of a car manufacturing enterprise (J Company) and studies storage between the paint shop and assembly shop. Considering the existing heuristic sequencing rules, the sequencing algorithm which combines intelligent algorithm with heuristic algorithm is used to deal with the storage sequencing problems. A two-phase optimization algorithm is proposed based on the small-world theory and the heuristic method. This algorithm realizes the optimization of the production target in the assembly shop with the reordering of the vehicle sequence through the painting storage.

\section{The Painted Body Storage Sequencing Problem}

In the mixed-model assembly line of automobile, the TAKT time of the paint shop is usually inconsistent with that of the assembly shop. The painted body storage is therefore set up to adjust the sequence of different painted bodies and keep pace between the two shops. The main task of the paint shop is to paint the white body. In order to reduce the paint cleaning loss of frequent color change, the times of color switching need to be minimized for the vehicles going into the paint shop. There is no special requirement to the color for the assembly shop. However, the consumption rate of the key parts and the production load for each workstation are required to be emphasized. Therefore, the sequence of vehicle going into the assembly shop through the PBS needs to be reordered to realize the optimization of the production objective in the assembly shop.

2.1. Description of PBS. Wortmann and Spieckermann [19] summarized various storage forms in the production system. According to the structure and function of storage, storage can be divided into list storage, stack storage, circular storage, linear storage, retroposition storage, and free storage. These storages differ in sequencing capacity, equipment cost and maintenance, and so forth.

This paper studies a multichannel linear storage. Figure 1 shows the structure diagram. To simplify the description of the multichannel linear storage model, the process of products getting into the multichannel storage is called inbound, and the process of products getting out of the multichannel storage is called outbound. The FIFO queue is called channel. The multichannel storage is to alleviate the inconsistence of the cycle time in the upstream and downstream production. There is a certain amount of WIP in the practical production. The storage sequencing is also a process with dynamical continuous input and output products. In order to simplify the calculation, the dynamical problem is converted into the static problem, and the continuous production process becomes the problem with multiple production batches. Then the storage sequencing for each batch becomes a static problem.

Linear storage has two essential parameters: the capacity of storage $V$ and the number of sequencing channels $N$. Assume that $L$ presents the length of the channel; then $V=$ $L \times N$. The optimization sequencing problem of PBS is described as follows: through a multichannel storage $\Xi=$ $(V, N)$, for a work in process (WIP) sequence with the length $n$, the sequence of the upstream shop $w$ is translated into the sequence $w^{\prime}$ with the optimization of the objective $f$ in the downstream shop. $f$ is (1) and (2) in Sections 2.2 and 2.3 , in order to minimize the leveling of the logistic cost and production load balance for each station in the downstream shop. The following are the problem assumptions.

$w$ : it means the sequence which consists of $n$ WIP in the upstream shop. The $i$ th WIP could be the same type with the $j$ th. 


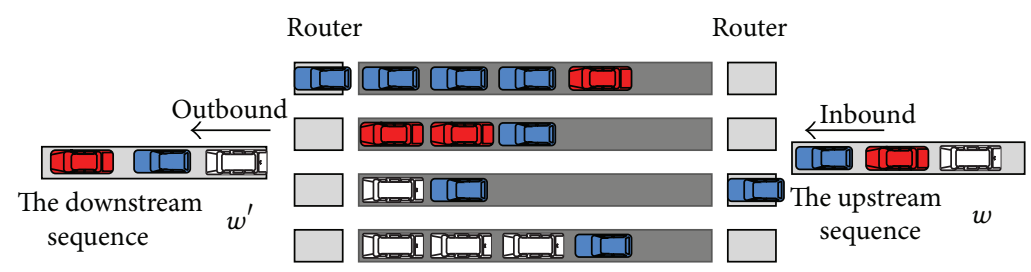

FIgURE 1: The structure diagram linear storage.

$w^{\prime}$ : it means the sequence which consists of $n$ WIP in the downstream shop. The $i$ th WIP could be the same type with the $j$ th.

$\Xi$ : it is the linear storage; $\Xi=(V, N)$. $V$ is the capacity of the storage and $N$ is the number of the channels into which the products could enter.

For all the above, $i, j=1,2, \ldots, n$.

The objective of PBS sequencing problem is minimizing the consumption rate of options, the total overtime, and the idle time of workstations when assigning $n$ vehicles into the multichannel storage. According to the description of PBS, the size of the problem depends on the sequence length of WIP $n$ and the number of the channels $N$; that is, $\left(C_{N}^{1}\right)^{n} \times$ $\left(C_{N}^{1}\right)^{n}=N^{2 n}$. With the increasing of the product sequence length, the size of the problem increases exponentially. So the problem is also a NP-hard problem.

In the practical application, the heuristic method based on rules is always used to solve this problem [20]. Considering the production sequencing process of J Company, the common inbound heuristic rules are as follows according to the order of priority from high to low.

Rule 1. If the material requirement of an inbound product is consistent with the last product in the channel, the product will go into the channel.

Rule 2. If the channel meeting rule 1 cannot be found, an empty channel will be chosen.

Rule 3. If the channel meeting rule 2 cannot be found, the channel with the least WIP will be chosen.

The common outbound heuristic rules are as follows.

Rule 1. Select the WIP in which the material requirement is inconsistent with the previous outbound WIP.

Rule 2. If the channels meeting rule 1 cannot be found, a WIP from the channel with the most WIP will be selected.

Based on these rules, similar products should be put together for inbound to simplify the subsequent sequencing process. The outbound rules consider the objective of the assembly shop. The material distribution should be leveled in the assembly shop, in order to balance the production load for each workstation. This paper quantifies the abstract rules; then PBS problem can be converted to an optimization sequencing problem which will realize the leveling of material consumption and the balancing of station load.
2.2. Minimizing the Consumption Rate of Options. Considering the assembly shop objectives, we use one function described in Miltenburg [21] and used in numerous works $[22,23]$. Cycle sorting method is often used to balance the output ratio of product in the plan sequencing of mixedmodel assembly enterprise [24]. Based on the bill of materiel (BOM) of the master production plan, the BOM of each product can be calculated based on the layered and the calculation method of material requirements planning (MRP). The model parameters are given as follows:

$n$ : number of vehicles in a sequence,

$I$ : number of options of the PBS,

$m_{i}$ : number of options of type $i$ to produce, $i=$ $1,2, \ldots, I$

$W_{i}$ : weight of the option $i, i=1,2, \ldots, I$,

$p$ : position of the vehicle, $p=1,2, \ldots, n$,

$x_{i, p}$ : number of vehicles with the option $i$ between the positions 1 and $p, x_{i, p}=0,1, \ldots, n-1$.

The minimization of the consumption rate of options is

$$
f_{1}=\sum_{p=1}^{n} \sum_{i=1}^{I} \frac{W_{i}\left(\left(x_{i, p} / p\right)-\left(m_{i} / n\right)\right)^{2}}{\sum_{i=1}^{I} W_{i}} .
$$

$f_{1}$ denotes the smoothing function for the assembly shop by calculating the outbound sequence. $f_{1}$ is an objective function measuring the variability in the resource usage.

2.3. Minimizing Total Overtime and Idle Time of Workstations. As the diversified vehicles have different demands, the workstations in the case company are often in the status of overload or idle. The switching time between different vehicles is ignored here. The workstation is idle when an operator finished the operation on a vehicle and is waiting for the next vehicle on the workstation. The workstation is overload if a new vehicle arrives in the station before the operator finishes processing the previous vehicle on the station and the remaining work must be processed in time by another worker outside the workstation $[25,26]$. In order to balance the processing time of each workstation in the whole mixed-model assembly line, it is important to optimize 
the overload and idle time of workstations. The optimization model is established as follows:

$$
\begin{gathered}
f_{2}=\min \left\{\sum_{j=1}^{J} \sum_{i=1}^{I}\left(\mathrm{idt}_{j i}+\mathrm{ovt}_{j i}\right)\right\} \\
\text { s.t. } \quad \sum_{m=1}^{M} x_{m i}=1 \quad \forall i \\
x_{m i}=0 \text { or } 1 \quad \forall m, i m=1,2, \ldots, M \\
S_{j 1}=0 \quad j=1,2, \ldots, J \\
E_{j i}=S_{j i}+T_{j m} \\
S_{j i+1}=\max \left\{0, E_{j i}-C\right\} \\
\text { idt }_{j i}=\max \left\{0, C-E_{j i}\right\} \\
\text { ovt }_{j i}=\max \left\{0, E_{j i}-C\right\} .
\end{gathered}
$$

$x_{m i}$ is 1 if the $i$ th vehicle of the assembly sequence belongs to type $m$; otherwise $x_{m i}$ is $0 . S_{j i}$ indicates the start time when $i$ th vehicle is at the $j$ th station. $E_{j i}$ represents the end time when $i$ th vehicle is at $j$ th station. $C$ is the cycle time of the mixed-model assembly line. $\mathrm{idt}_{\mathrm{ji}}$ and $\mathrm{o} v \mathrm{t}_{\mathrm{ji}}$ represent the idle time and the overload time of $i$ th vehicle on the $j$ th station, respectively.

\section{A Two-Phase Algorithm for the PBS Sequencing}

Since the sequence of the painted body first goes into the storage and then goes out of the storage, the algorithm is divided into two phases as well, the inbound algorithm and the outbound algorithm. By using the DSWOA and the heuristic algorithm, there are four combinations of the algorithm:

(1) the inbound heuristic algorithm and the outbound heuristic algorithm,

(2) the inbound heuristic algorithm and the outbound DSWOA,

(3) the inbound DSWOA and the outbound heuristic algorithm,

(4) the inbound DSWOA and the outbound DSWOA.

Combination 1 is widely applied in practice, also being used in the case company. In combination 2, the problem is simplified as a job-shop scheduling problem and the results depend on the selection of the heuristic algorithm; the DSWOA has a limited solution space. In combination 4, the two-phase DSWOA costs much more time and is seldom applied in practice.

Due to the above reasons, combination 3 is selected to solve the model. This method can give more optimization space to the DSWOA and closely combine the objective function to the outbound rules. For any inbound vehicle sequence $w$, we can get the outbound vehicle sequence $w^{\prime}$. Thus, the PBS sequencing problem is converted into a twophase optimization process: optimizing the inbound routing strategy by DSWOA and scheduling the outbound sequence by heuristic algorithm.

3.1. DSWOA for Inbound Process Optimization. In the DSWOA, each solution under consideration is called an envelope node. The basic ideas of small-world optimization are described as follows: first deliver several envelopes from different places in the original solution space and regard each envelope node as a candidate solution, then inquire each envelope node's short-range and long-range contacts nodes in the solution space, deliver the envelope to the contacts node which has a better objective value, and at last, each envelope will be closer to the objective node through the delivery in the solution space of small-world network. In summary, the optimization of DSWOA is an iterative process that envelope nodes deliver their envelopes to their contacts nodes. The computation steps of the DSWOA are as follows.

(1) Set the parameters, the number of the envelope nodes, the number of short-range and long-range contacts nodes, the iteration times, and so forth.

(2) Initialize the population. Randomly generate a certain number of solutions (or envelope nodes).

(3) Inquire the neighbor nodes. A certain number of short-range and long-range contacts nodes are inquired for each envelope node in the solution space.

(4) Evaluate the inquired contacts nodes of each envelope node. Calculate the objective value for each contacts node, and sort them.

(5) Update envelope nodes. Select the best contacts node to replace the current envelope node. Calculate all the selected contacts nodes and select the node with the best objective value.

(6) If the termination criterion is reached, return the best solution found so far; otherwise go to step 3.

3.1.1. Encoding and Initialization. The optimization for PBS sequencing problem is solved by a two-phase optimization process. The vehicle sequence in upstream paint shop is coded as $w=\{1,2, \ldots, n\}$ by integers, where $n$ is the total number of vehicles to sequence. In the SWO algorithm, supposing $m$ is the number of envelopes and $n$ is the coding length, for each node, the inbound routing solution is represented by a permutation of jobs as $X_{i}=\left\{x_{i 1}, x_{i 2}, \ldots, x_{i j}, \ldots, x_{i n}\right\}, i=$ $1,2, \ldots, m, j=1,2, \ldots, n$, for all $x_{i j}=1,2, \ldots, N$, where $m$ indicates the number of envelope nodes, $N$ represents the number of PBS channel, and $x_{i j}$ is the selected inbound channel of production $j$ in the envelope node $i$.

The initial cluster $X_{i}$ is composed of the original mail holders, and each node represents a candidate solution. The initial cluster is randomly generated as follows:

$$
x_{i j}=\text { Math.Ceil }(\text { Random }() \times N) \text {. }
$$


As the routing solution $X_{i}$ cannot directly indicate the real situation in the PBS, we introduce a variable $P B S_{-} X_{a b}$ to indicate the vehicle information of position $b$ in the channel $a$ for PBS. If $P B S_{-} X_{a b}=0$, there is no vehicle in this position. $X_{i}$ can be transformed into $P B S \_X_{a b}$ by a short procedure; the following example illustrates the transformation.

Assuming that the number of total vehicles is 15 , the number of PBS channel is 4 . Randomly generate an inbound routing strategy $X=\{2,2,2,1,1,3,3,3,3,3,4,4,1,1,1\}$; then the vehicles in the PBS can be described as in Figure 2 and the sequence in PBS can be indicated as

$$
P B S_{-} X=\left\{\begin{array}{ccccccc}
4 & 5 & 13 & 14 & 15 & 0 & \cdots \\
1 & 2 & 3 & 0 & 0 & 0 & \cdots \\
6 & 7 & 8 & 9 & 10 & 0 & \ldots \\
11 & 12 & 0 & 0 & 0 & 0 & \ldots
\end{array}\right\}
$$

In this example, the optimization for the inbound routing strategy is transformed into the optimization for the vehicle sequence in PBS channel and the optimization for PBS $X$ is more intuitive. At the same time, the problem of optimizing the $P B S \_X$ is very similar to the vehicle routing problem (VRP); the main difference is that the sequence in each PBS channel should be in an ascending order. From the structure of the PBS, switching all the vehicles between any two channels will not change the outbound sequence.

3.1.2. The Short-Range and Long-Range Operators. The distinctive feature of small-world network is the presence of large amounts of short neighbor relations. The small-world network models proposed by Watts and Strogatz [12] and Kleinberg [27] defined the short-range and long-range contacts for each individual. In the DSWOA, these two contacts are redefined to search for the optimization objectives.

In the small-world network models proposed by Watts and Strogatz [12] and Kleinberg [27], there is small difference between the short-range nodes; however the difference between the long-range nodes becomes larger. For the sequence coding in each channel of PBS shown in Figure 2, the operation that will not change the number of vehicles in each channel is defined as a short-range operator, as shown in Figure 3. If the number of vehicles in all channels has changes, the operation is called long-range operator, such as the two methods described in Figure 4. It should be noted that the vehicle sequence in PBS may be an illegal solution after the short-range and long-range operator. To ensure the sequence is shorter than the length of PBS channel, the check method is needed to change the length of vehicle sequence in every channel and rearrange the vehicle sequence in ascending order.

Operator $M_{1}$ selects several elements in any two channels to swap. Operator $M_{2}$ exchanges all the elements after some position in any two channels. Operator $M_{3}$ inserts an element of a sequence before some position of another sequence. Operator $M_{4}$ inserts a string of elements of a sequence before some position of another sequence. The vehicle sequence after all the operations should be rearranged in ascending order.

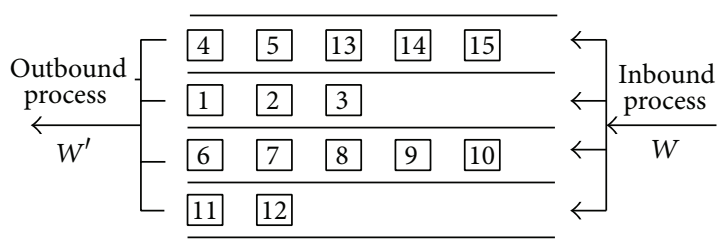

Figure 2: An example of the inbound routing strategy.

\begin{tabular}{|c|c|}
\hline $\begin{array}{lllll}4 & 5 & 13 & 14 & 15 \\
\end{array}$ & 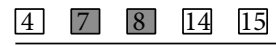 \\
\hline \begin{tabular}{|lll}
1 & 2 & 3 \\
\end{tabular} & 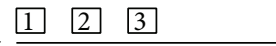 \\
\hline \begin{tabular}{|l|l|l|l|l}
6 & 7 & 8 & 9 & 10 \\
\end{tabular} & $\begin{array}{lllll}5 & 6 & 9 & 10 & 13 \\
\end{array}$ \\
\hline 1112 & $11 \quad 12$ \\
\hline \multicolumn{2}{|c|}{$M_{1}$ (crossover operator) } \\
\hline \begin{tabular}{|lllll}
4 & 5 & 13 & 14 & 15 \\
\end{tabular} & \begin{tabular}{|l|l|lll|}
4 & 5 & 13 & 14 & 15 \\
\end{tabular} \\
\hline \begin{tabular}{|l|l|l|}
1 & 2 & 3 \\
\end{tabular} & \begin{tabular}{|lllll}
1 & 2 & 8 & 9 & 10 \\
\end{tabular} \\
\hline \begin{tabular}{|l|l|l|l|l|}
6 & 7 & 8 & 9 & 10 \\
\end{tabular} & \begin{tabular}{|l|l|l|}
3 & 6 & 7 \\
\end{tabular} \\
\hline \begin{tabular}{|l|l|}
11 & 12 \\
1
\end{tabular} & \begin{tabular}{|l|l|}
11 & 12 \\
1
\end{tabular} \\
\hline
\end{tabular}

Figure 3: Two kinds of short-range operators.

3.1.3. Updating the Envelope Node. In the iterative process of DSWO, the envelope nodes need to be updated in each generation. When delivering the envelope, we select a fixed number of short-range contacts nodes generated by the short-range operators $M_{1}$ and $M_{2}$, as well as some longrange contacts nodes by long-range operators $M_{3}$ and $M_{4}$ in Section 3.1.2. Finally, we compare the selected nodes with the envelope node by calculating the objective values of these nodes. The envelope node with better objective value replaces the current node.

3.2. Heuristic Method for Outbound Process Optimization. Several common outbound rules for PBS sequencing problem are introduced in Section 2.1. The rule-based heuristic method is featured by the simplicity and rapidity and it conforms to the production sequencing operators' experience guidance method; it gains a wide application in practice. The heuristic method in the J vehicle company mainly considers the component properties of the vehicle sequence in each channel, avoiding the vehicles with the same parts demand for going out of the storage continuously.

The objective function in Section 2.2 shows that the total number of each option demand $n_{i}$ is calculated by the BOM of WIP; when vehicle $k$ is outbound, the consumption rate of options can be defined as

$$
f_{k}=\sum_{p=1}^{k} \sum_{i=1}^{I} \frac{W_{i}\left(\left(x_{i, p} / p\right)-\left(n_{i} / M\right)\right)^{2}}{\sum_{i=1}^{I} W_{i}},
$$

where $x_{i, k}$ is the number of demanded parts. For a PBS with $N$ channels, the channel with the smallest consumption rate will be chosen to outbound the vehicle. 


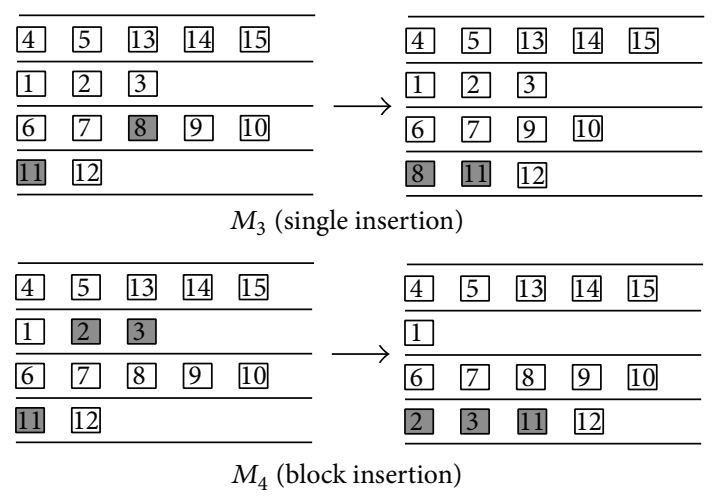

FIgURE 4: Two kinds of long-range operators.

3.3. The Procedure of the Two-Phase Algorithm. The DSWOA has a superior searching and updating strategy when solving the PBS sequencing problem. After searching for the shortrange and long-range neighbors, an ideal target node will be found through continuous delivery and iteration. The DSWOA is only used to optimize the inbound sequence. The outbound operation is optimized by the greedy algorithm, providing a clear target for the inbound optimization. The combination of the two algorithms realizes the optimization of the PBS sequencing problem. The flow chart of the algorithm is shown in Figure 5.

\section{Computational Experiments and Results}

The J Company produces multipurpose vehicles, trucks, and so forth. This paper mainly studies the sequencing problem of the painted body storage in a multipurpose vehicles production line. The WIP undergoes spray-painting in the painted shop and the TAKT time is the longest in all workstations, so the storage is particularly necessary. In order to minimize the consumption rate of storage option and the sum of overtime and idle time of the workstations, we use the DSWOA method and heuristic rules to schedule the inbound and outbound sequence.

A number of numerical experiments are conducted using the actual production data in the J Company to compare the DSWOA with the genetic algorithms for the PBS sequencing problem. The algorithm is realized in Java and the experiments are performed on a PC with Intel Core $2.53 \mathrm{GHZ}$.

4.1. Preparing the Actual Production Data. To simplify the calculation, this paper focuses on the critical options and the key workstations. In the master production plan, one batch includes 40 vehicles and there are 12 different kinds of vehicles in one batch, as is shown in Table 1. Each vehicle is made up of 6 critical parts: engine, front axle, rear axle, gearbox, wheel, and braking system. Different types of vehicles can have the same type of critical part; for example, vehicle model $a$ and vehicle model $f$ have the same type of engine. Moreover, each type of vehicle has similar processes and we focus on 10 critical processes. The times of each process for different

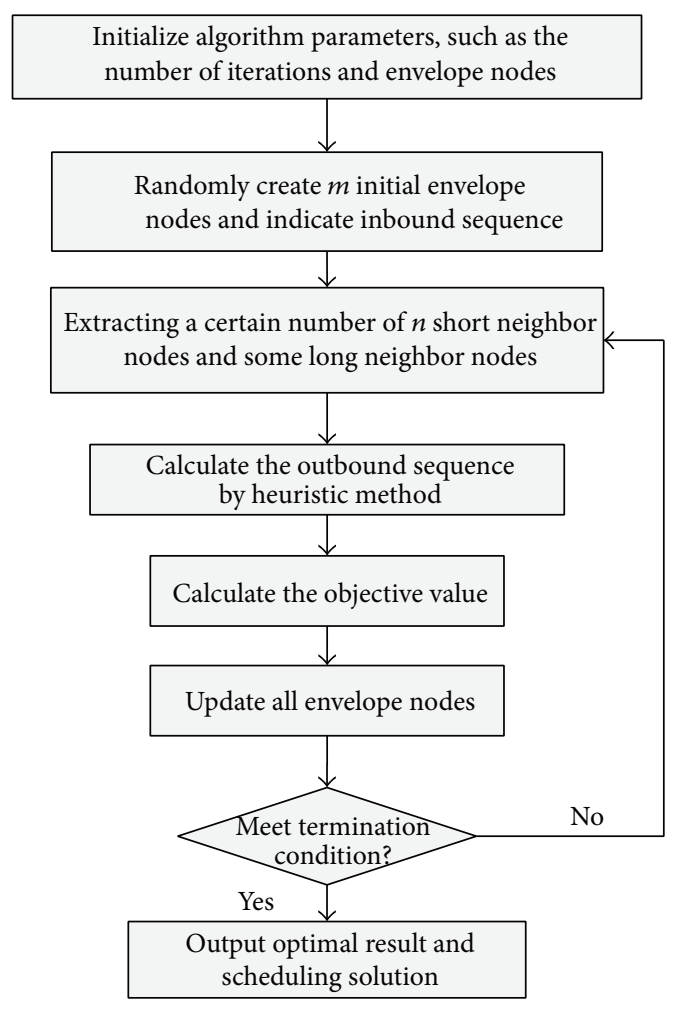

FIGURE 5: The flow chart of the two-phase algorithm for PBS sequencing problem.

models of vehicle are shown in Table 2. For example, the first process of vehicle model $a$ needs 105 seconds.

According to the difference of throughput and production TAKT time between the painted shop and assembly shop, we designed a PBS with 5 channels and each channel can hold 16 vehicles. The PBS is denoted by $\Xi=(80,5)$.

4.2. Resolution and Results Analysis of the Case Problem. According to the major plan, each production batch contains 12 kinds and 40 vehicles. The original sequence in one batch is

$$
\text { aaaabbbcccccddeeefgggggghhhiiiijjkkkkkkl. }
$$

We code the sequence by integer as is shown in Table 3 .

In the DSWOA's optimization process of the multiobjective PBS sequencing problem, for the target of each envelope nodes Goal ${ }_{i}=\left(f_{1, i}, f_{2, i}\right)$, only when $f_{1, i+1}<f_{1, i}, f_{2, i+1}<f_{2, i}$, the envelope nodes updates, $\operatorname{Goal}_{i+1}=\left(f_{1, i+1}, f_{2, i+1}\right)$.

The parameters in SWO are set as follows: the number of envelope nodes is 50; each envelope node selects 20 shortrange nodes and 5 long-range nodes; the iteration is 100.

4.2.1. The Consumption Rate of Options. Based on the data in Tables 1 and 2, the ideal consumption rate of each option is calculated and the results are shown in Table 4.

Based on the heuristic rules for inbound and outbound in PBS sequencing process mentioned in Section 2.1, the outbound sequence is calculated as 
TABLE 1: The major plan of actual production data and critical parts of each product model.

\begin{tabular}{|c|c|c|c|c|c|c|c|}
\hline Vehicle model & Amount & Engine & Front axle & Rear axle & Gearbox & Wheel & Braking system \\
\hline $\mathrm{a}$ & 4 & S1-3 & S2-1 & S3-1 & S4-1 & S5-1 & S6-1 \\
\hline b & 3 & S1-2 & S2-2 & S3-2 & S4-2 & S5-2 & S6-2 \\
\hline c & 5 & S1-2 & $\mathrm{S} 2-1$ & S3-1 & S4-3 & S5-2 & S6-2 \\
\hline d & 2 & S1-1 & S2-2 & S3-1 & S4-2 & S5-2 & S6-1 \\
\hline e & 3 & S1-4 & $\mathrm{S} 2-1$ & S3-2 & S4-1 & S5-2 & S6-3 \\
\hline $\mathrm{f}$ & 1 & S1-3 & S2-1 & S3-1 & S4-3 & S5-1 & S6-3 \\
\hline g & 6 & S1-4 & S2-2 & S3-1 & S4-1 & S5-2 & S6-1 \\
\hline $\mathrm{h}$ & 3 & S1-1 & $\mathrm{S} 2-1$ & S3-2 & S4-1 & S5-1 & S6-2 \\
\hline $\mathrm{i}$ & 4 & S1-1 & S2-1 & S3-1 & S4-2 & S5-2 & S6-2 \\
\hline j & 2 & S1-2 & S2-2 & S3-1 & S4-3 & S5-1 & S6-1 \\
\hline $\mathrm{k}$ & 6 & S1-2 & S2-1 & S3-2 & S4-2 & S5-2 & S6-3 \\
\hline 1 & 1 & S1-4 & S2-2 & S3-2 & S4-3 & S5-1 & S6-2 \\
\hline
\end{tabular}

TABLE 2: The processing time in each critical workstation for 12 kinds of vehicles.

\begin{tabular}{lccccccccccc}
\hline $\begin{array}{l}\text { Vehicle } \\
\text { model }\end{array}$ & T01 & T02 & T03 & T04 & T05 & T06 & T07 & T08 & T09 & T10 \\
\hline $\mathrm{a}$ & 105 & 113 & 90 & 105 & 108 & 109 & 101 & 94 & 109 & 104 \\
$\mathrm{~b}$ & 115 & 107 & 100 & 107 & 110 & 113 & 104 & 97 & 108 & 114 \\
$\mathrm{c}$ & 112 & 106 & 103 & 94 & 115 & 95 & 106 & 109 & 113 & 97 \\
$\mathrm{~d}$ & 107 & 115 & 113 & 105 & 107 & 112 & 103 & 100 & 106 & 107 \\
$\mathrm{e}$ & 90 & 112 & 105 & 116 & 113 & 96 & 109 & 103 & 102 & 93 \\
$\mathrm{f}$ & 120 & 112 & 105 & 106 & 114 & 117 & 106 & 90 & 112 & 121 \\
$\mathrm{~g}$ & 97 & 108 & 112 & 99 & 107 & 113 & 95 & 102 & 107 & 97 \\
$\mathrm{~h}$ & 108 & 99 & 105 & 113 & 104 & 93 & 107 & 110 & 113 & 99 \\
$\mathrm{i}$ & 104 & 106 & 93 & 114 & 109 & 110 & 115 & 93 & 112 & 92 \\
$\mathrm{j}$ & 101 & 104 & 115 & 117 & 94 & 103 & 112 & 97 & 99 & 105 \\
$\mathrm{k}$ & 112 & 104 & 105 & 99 & 115 & 103 & 102 & 93 & 107 & 113 \\
$\mathrm{l}$ & 105 & 121 & 116 & 98 & 104 & 101 & 113 & 94 & 107 & 99 \\
\hline
\end{tabular}

1526374891011153216331719123413203514 2136182822372529233826304024392731

which corresponds to the practical outbound sequence abababaccccejejegckdgkdgkfigkhigkhilgkhi.

We can get the consumption rate goal $f_{1}=7.8923$.

When only taking into account the goal of minimizing the consumption rate of options, we can get an optimal solution calculating by the two-phase algorithm. The outbound sequence is

4191551661421181722731343883209102329 3637401322112512172628303224333539

and the corresponding practical outbound sequence is agebebdgfabahikkcagccgikkldgchcehiijgjkk.

The consumption rate goal is $f_{1}=0.2714$ and the vehicle sequence in the PBS channel is shown in Table 5.

Considering the objective of the consumption rate of options, the result calculated by the two-phase algorithm is

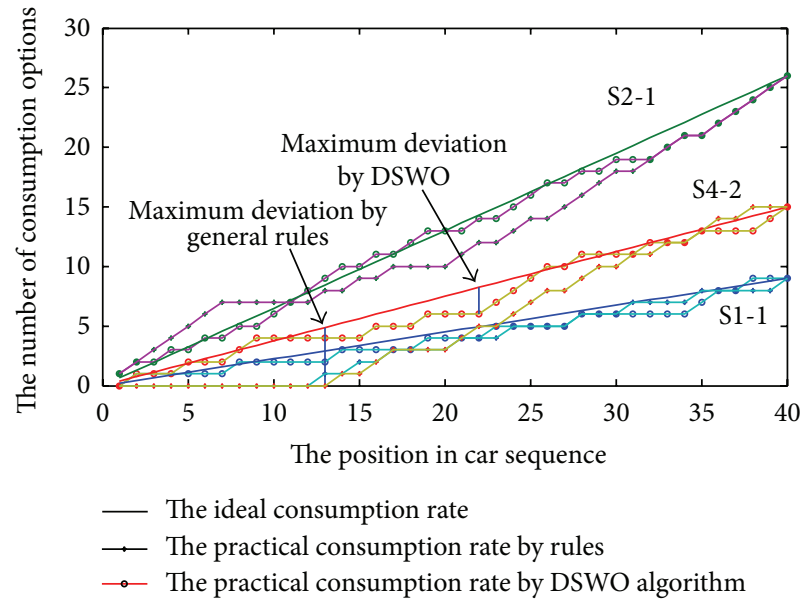

FIGURE 6: The comparison chart between the practical consumption rate and ideal consumption rate for three options.

the $3.4 \%$ of that by the heuristic rules, so the two-phase algorithm performs better.

In order to fully demonstrate the effectiveness of the two-phase algorithm, we select three kinds of parts (S11, S2-1, and S4-3) which urgently demand to analyze the difference between the practical consumption rate and the ideal consumption rate. In the consumption chart shown in Figure 6, the practical consumption rate is very close to the ideal consumption rate by using two-phase algorithm and the maximum deviation is 2.25 ; however, the practical consumption rate has a great difference with that by using heuristic rules and the maximum deviation is 4.875 . It can be found that the two-phase algorithm can effectively solve the problem of minimizing the consumption rate of options and constrain the fluctuation of the consumption rate of the options. Therefore, the optimization solution could be used to guide production in practice.

4.2.2. The Pareto Solutions Set of the Multiobjective PBS Sequencing Problem. DSWOA is proven for its favorable searching capability in the previous section. In this section, 
TABLE 3: The coding vehicle sequence.

\begin{tabular}{lccccccccccccccccccccc}
\hline Vehicle model & $\mathrm{a}$ & $\mathrm{a}$ & $\mathrm{a}$ & $\mathrm{a}$ & $\mathrm{b}$ & $\mathrm{b}$ & $\mathrm{b}$ & $\mathrm{c}$ & $\mathrm{c}$ & $\mathrm{c}$ & $\mathrm{c}$ & $\mathrm{c}$ & $\mathrm{d}$ & $\mathrm{d}$ & $\mathrm{e}$ & $\mathrm{e}$ & $\mathrm{e}$ & $\mathrm{f}$ & $\mathrm{g}$ & $\mathrm{g}$ \\
Integer coding & 1 & 2 & 3 & 4 & 5 & 6 & 7 & 8 & 9 & 10 & 11 & 12 & 13 & 14 & 15 & 16 & 17 & 18 & 19 & 20 \\
\hline Vehicle model & $\mathrm{g}$ & $\mathrm{g}$ & $\mathrm{g}$ & $\mathrm{g}$ & $\mathrm{h}$ & $\mathrm{h}$ & $\mathrm{h}$ & $\mathrm{i}$ & $\mathrm{i}$ & $\mathrm{i}$ & $\mathrm{i}$ & $\mathrm{j}$ & $\mathrm{j}$ & $\mathrm{k}$ & $\mathrm{k}$ & $\mathrm{k}$ & $\mathrm{k}$ & $\mathrm{k}$ & $\mathrm{k}$ & $\mathrm{l}$ \\
Integer coding & 21 & 22 & 23 & 24 & 25 & 26 & 27 & 28 & 29 & 30 & 31 & 32 & 33 & 34 & 35 & 36 & 37 & 38 & 39 & 40 \\
\hline
\end{tabular}

TABLE 4: The ideal consumption rate of each option.

\begin{tabular}{lcc}
\hline Option number & Total demand & The ideal consumption rate \\
\hline S1-1 & 9 & 0.225 \\
S1-2 & 16 & 0.400 \\
S1-3 & 5 & 0.125 \\
S1-4 & 10 & 0.250 \\
S2-1 & 26 & 0.650 \\
S2-2 & 14 & 0.350 \\
S3-1 & 24 & 0.600 \\
S3-2 & 16 & 0.400 \\
S4-1 & 16 & 0.400 \\
S4-2 & 15 & 0.375 \\
S4-3 & 9 & 0.225 \\
S5-1 & 11 & 0.275 \\
S5-2 & 29 & 0.725 \\
S6-1 & 14 & 0.350 \\
S6-2 & 16 & 0.400 \\
S6-3 & 10 & 0.250
\end{tabular}

TABle 5: The vehicle sequence in the PBS channel.

\begin{tabular}{lccccccccccc}
\hline Channel 1 & 4 & 15 & 16 & 27 & 31 & 34 & 38 & & & & \\
Channel 2 & 19 & 21 & 23 & 29 & 36 & 37 & 40 & & & & \\
Channel 3 & 1 & 2 & 3 & 9 & 10 & 13 & 25 & & & & \\
Channel 4 & 14 & 18 & 20 & 22 & 26 & 28 & 30 & 32 & & & \\
Channel 5 & 5 & 6 & 7 & 8 & 11 & 12 & 17 & 24 & 33 & 35 & 39 \\
\hline
\end{tabular}

the DSWOA is compared with genetic algorithm. The parameters of GA are set as follows: the size of population is 50; the genetic crossover probability is 0.4 ; the gene mutation probability is 0.1; the iteration number is 100 .

By calculating the two objectives of the PBS sequencing problem, we obtain a set of Pareto solutions by DSWOA and GA as shown in Figure 7. It can be found that the Pareto solutions by the DSWOA is proved better than the GA. It proves that the DSWOA has a comparable performance with the GA and in some ways even better than GA.

4.2.3. The Influence of PBS Parameters. The channel number and the storage capacity greatly affect the optimization result. When the channel number is 1 , the storage cannot schedule the upstream inbound sequence. When the channel number is infinite, the PBS sequencing problem can be regarded as a common vehicle sequencing problem.

In order to investigate the impact of the channel number and the capacity of storage for the solution, we only consider the objective of minimizing the consumption rate of options.

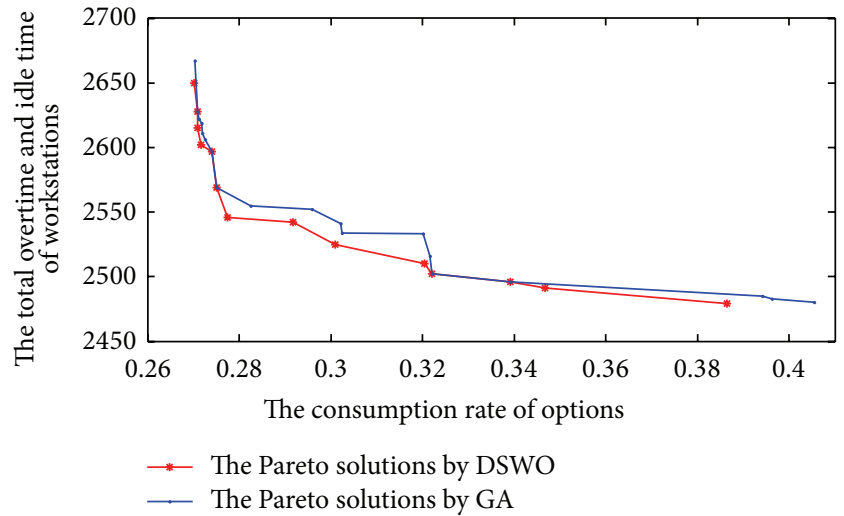

FIgURE 7: The comparison chart of Pareto solution between the DWSO algorithm and GA.

This paper designs a set of the following contrast experiments as in Table 6.

In Table 6, it is obvious that when the number of storage channel gradually increases, the optimization results of the consumption rate of options decrease. When the number of storage channel reaches a certain value, the objective achieves the optimal value and increasing the channel number is meaningless. We also find that when only changing the length of the storage channel, the change of the target value is insignificant. Therefore, the channel number is an important parameter in the designing process of storage capacity; the channel length only needs to meet the requirement of storage capacity.

\section{Conclusion}

To improve the efficiency of PBS, we develop a mathematical model aiming at minimizing the consumption rate of options and the sum of overtime and idle time of workstations. As the PBS sequencing contains upstream sequence inbound and downstream sequence outbound. The two-phase algorithm includes the DSWOA and HA, is proposed. The mathematical model and the two-phase algorithm are applied in a PBS of a vehicle manufacturing enterprise. The results indicate that the two-phase algorithm is suitable for the PBS sequencing problem and the DSWOA has a better searching ability than GA in this problem. At last, we analyze the sensitivity of the parameters of PBS and conclude that the channel number is an important factor in the designing process of storage capacity.

This research provides a new method to solve the PBS sequencing problem. However, the practical PBS sequencing problem is a dynamical problem. Further research is needed 
TABLE 6: The consumption rate of options under different paremeters of PBS.

\begin{tabular}{|c|c|c|c|c|}
\hline Test number & Channel number & Channel length & Average consumption rate of 10 experiment & The best solution \\
\hline 1 & \multirow{3}{*}{3} & 15 & 0.3394 & 0.3435 \\
\hline 2 & & 20 & 0.3349 & 0.3452 \\
\hline 3 & & 30 & 0.3359 & 0.3444 \\
\hline 4 & \multirow{3}{*}{4} & 10 & 0.2746 & 0.2997 \\
\hline 5 & & 15 & 0.2749 & 0.2859 \\
\hline 6 & & 20 & 0.2736 & 0.2929 \\
\hline 7 & \multirow{3}{*}{5} & 8 & 0.2705 & 0.2754 \\
\hline 8 & & 15 & 0.2713 & 0.2785 \\
\hline 9 & & 20 & 0.2711 & 0.2751 \\
\hline 10 & \multirow{3}{*}{6} & 7 & 0.2701 & 0.2718 \\
\hline 11 & & 10 & 0.2698 & 0.2727 \\
\hline 12 & & 15 & 0.2700 & 0.2729 \\
\hline 13 & \multirow{2}{*}{7} & 6 & 0.2693 & 0.2709 \\
\hline 14 & & 10 & 0.2695 & 0.2712 \\
\hline 15 & \multirow{2}{*}{8} & 5 & 0.2691 & 0.2705 \\
\hline 16 & & 10 & 0.2690 & 0.2709 \\
\hline 17 & 9 & 10 & 0.2690 & 0.2707 \\
\hline 18 & 10 & 10 & 0.2690 & 0.2704 \\
\hline 19 & 15 & 10 & 0.2690 & 0.2700 \\
\hline 20 & 20 & 10 & 0.2690 & 0.2700 \\
\hline 21 & 30 & 10 & 0.2690 & 0.2701 \\
\hline 22 & 40 & 10 & 0.2690 & 0.2703 \\
\hline
\end{tabular}

to find out better inbound and outbound methods in solving this problem. At the same time, when optimizing the PBS sequencing problem for Pareto solutions, this paper only considers the single object in/outbound operation. In the future research, the multiple objectives condition is worth being probed. Each objective could be transformed into a satisfactory function based on the actual requirements from industry.

\section{Conflict of Interests}

The authors declare that there is no conflict of interests regarding the publication of this paper.

\section{Acknowledgments}

The authors gratefully acknowledge the financial supports from the National Natural Science Foundation of China (nos. 51275191 and 51105157) and the Fundamental Research Funds for the Central Universities of HUST with the Grant no. 2014 TS033.

\section{References}

[1] H. Färber and C. A. M. Moreno, Overview on: Sequencing in Mixed Model Flowshop Production Line with Static and Dynamic Context, Universitat Politecnica De Catalunya, 2005.

[2] T. Epping and W. Hochstättler, Sorting with Line Storage Systems, Springer, New York, NY, USA, 2003.
[3] A. Jayaraman, R. Narayanaswamy, and A. K. Gunal, "A sortation system model," in Proceedings of the 29th Conference on Winter Simulation (WSC '97), pp. 866-871, IEEE Computer Society, 1997.

[4] A. Kavusturucu and S. M. Gupta, "Methodology for analyzing finite buffer tandem manufacturing systems with N-policy," Computers and Industrial Engineering, vol. 34, no. 4, pp. 837848, 1998.

[5] H. K. Aksoy and S. M. Gupta, "Capacity and buffer trade-offs in a remanufacturing system," International Society for Optics and Photonics, pp. 167-174, 2002.

[6] A. A. Bulgak, "Analysis and design of split and merge unpaced assembly systems by metamodelling and stochastic search," International Journal of Production Research, vol. 44, no. 18-19, pp. 4067-4080, 2006.

[7] E. Muhl, P. Charpentier, and F. Chaxel, "Optimization of physical flows in an automotive manufacturing plant: some experiments and issues," Engineering Applications of Artificial Intelligence, vol. 16, no. 4, pp. 293-305, 2003.

[8] S. Spieckermann, K. Gutenschwager, and S. Voß, "A sequential ordering problem in automotive paint shops," International Journal of Production Research, vol. 42, no. 9, pp. 1865-1878, 2004.

[9] D. H. Moon, C. Song, and J. H. Ha, "A dynamic algorithm for the control of automotive painted body storage," Simulation, vol. 81, no. 11, pp. 773-787, 2005.

[10] T. Braun, "Hungarian priority in network theory," Science, vol. 304, no. 5678, pp. 1745-1746, 2004.

[11] S. Milgram, “The small world problem," Psychology Today, vol. 2, no. 1, pp. 60-67, 1967. 
[12] D. J. Watts and S. H. Strogatz, "Collective dynamics of 'smallworld' networks," Nature, vol. 393, no. 6684, pp. 440-442, 1998.

[13] S. H. Strogatz, "Exploring complex networks," Nature, vol. 410, no. 6825 , pp. 268-276, 2001.

[14] T. Walsh, "Search in a small world," in Proceedings of the 16th International Joint Conference on Artificial Intelligence (IJCAI '99), vol. 2, pp. 1172-1177, Morgan Kaufmann Publishers, Stockholm, Sweden, August 1999.

[15] J.-Z. Chen, W. Liu, and J.-Y. Zhu, "Two-dimensional smallworld networks: navigation with local information," Physical Review E: Statistical, Nonlinear, and Soft Matter Physics, vol. 73, no. 5, Article ID 056111, 2006.

[16] Z. Cui, Y. Chu, and X. Cai, "Nearest neighbor interaction PSO based on small-world model," in Intelligent Data Engineering and Automated Learning-IDEAL 2009: Proceedings of the 10th International Conference, Burgos, Spain, September 23-26, 2009, vol. 5788 of Lecture Notes in Computer Science, pp. 633-640, Springer, Berlin, Germany, 2009.

[17] X. H. Li, J. H. Zhang, S. N. Wang, M. L. Li, and K. Li, "A small world algorithm for high-dimensional function optimization," in Proceedings of the IEEE International Symposium on Computational Intelligence in Robotics and Automation (CIRA '09), pp. 55-59, December 2009.

[18] J.-H. Li, X.-Y. Shao, Y.-M. Long, H.-P. Zhu, and B. R. Schlessman, "Global optimization by small-world optimization algorithm based on social relationship network," Journal of Central South University, vol. 19, no. 8, pp. 2247-2265, 2012.

[19] D. Wortmann and S. Spieckermann, "Manufacturing line simulation of automotive industry to enhance productivity and profitability," Automotive Simulation, vol. 95, pp. 91-106, 1995.

[20] S. Spieckermann, K. Gutenschwager, and S. Voß, "A sequential ordering problem in automotive paint shops," International Journal of Production Research, vol. 42, no. 9, pp. 1865-1878, 2004.

[21] J. Miltenburg, "Level schedules for mixed-model assembly lines in just-in-time production systems," Manage Science, vol. 35, no. 2, pp. 192-207, 1989.

[22] P. R. McMullen, "An ant colony optimization approach to addressing a JIT sequencing problem with multiple objectives," Artificial Intelligence in Engineering, vol. 15, no. 3, pp. 309-317, 2001.

[23] A. Joly and Y. Frein, "Heuristics for an industrial car sequencing problem considering paint and assembly shop objectives," Computers and Industrial Engineering, vol. 55, no. 2, pp. 295310, 2008.

[24] Y. Monden, “Toyota production system," Journal of the Operational Research Society, vol. 46, no. 5, pp. 669-670, 1995.

[25] A. Rahimi-Vahed and A. H. Mirzaei, "A hybrid multi-objective shuffled frog-leaping algorithm for a mixed-model assembly line sequencing problem," Computers \& Industrial Engineering, vol. 53, no. 4, pp. 642-666, 2007.

[26] Q. Liu, W.-X. Wang, K.-R. Zhu, C.-Y. Zhang, and Y.-Q. Rao, "Advanced scatter search approach and its application in a sequencing problem of mixed-model assembly lines in a case company," Engineering Optimization, vol. 46, no. 11, pp. 14851500, 2014.

[27] J. M. Kleinberg, "Navigation in a small world," Nature, vol. 406, no. 6798, p. 845, 2000. 


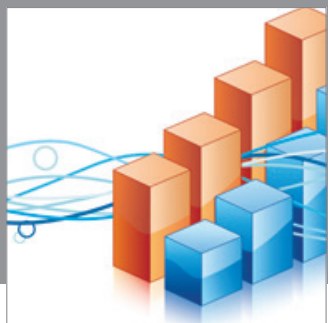

Advances in

Operations Research

mansans

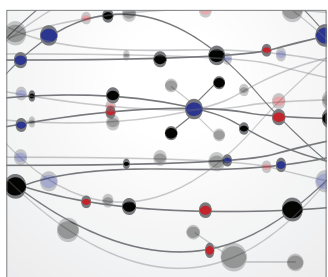

The Scientific World Journal
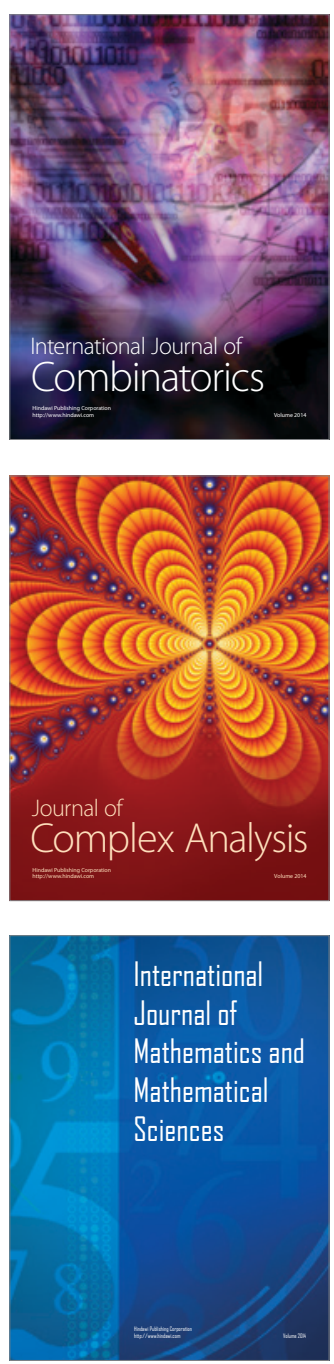
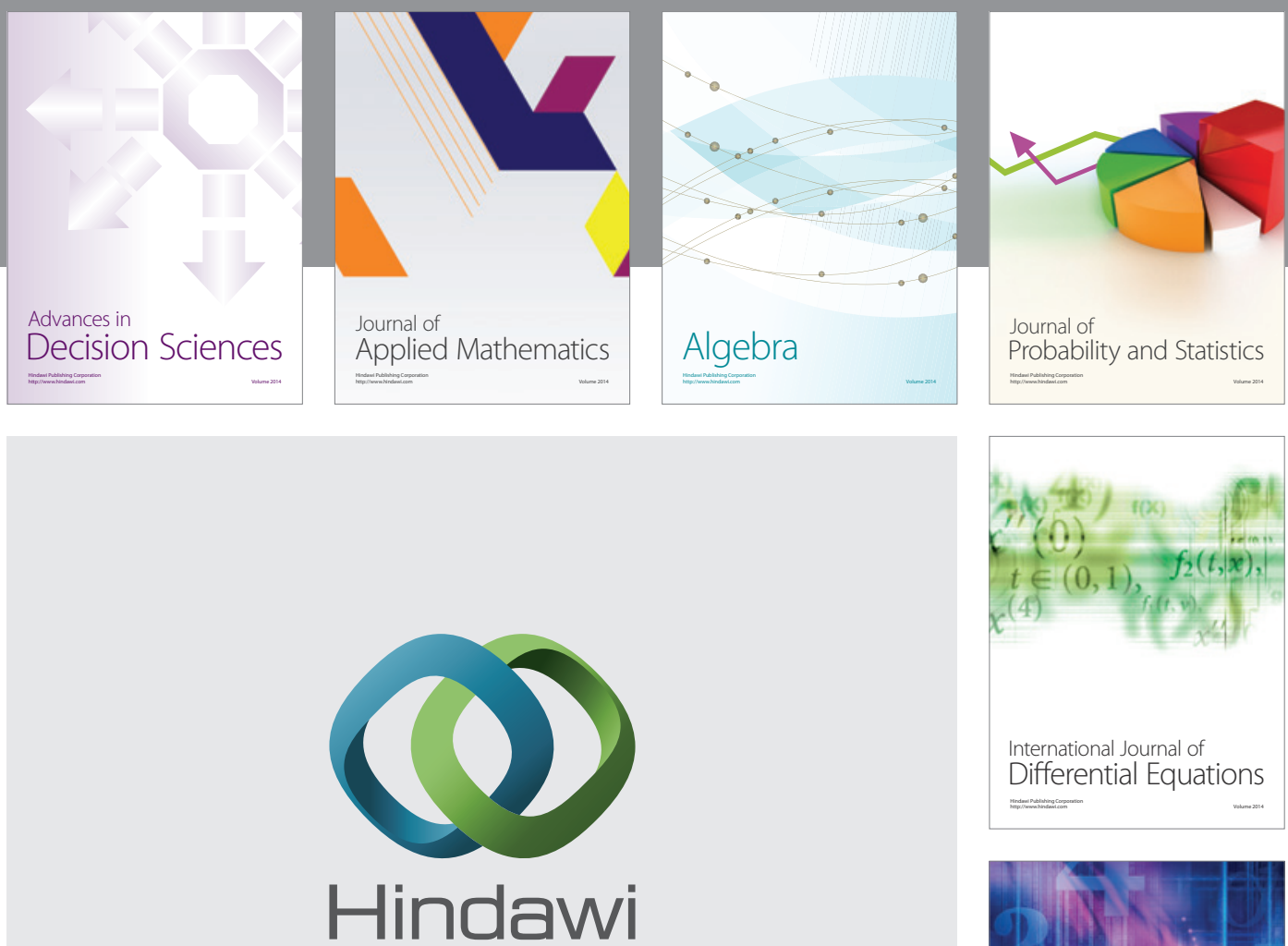

Submit your manuscripts at http://www.hindawi.com
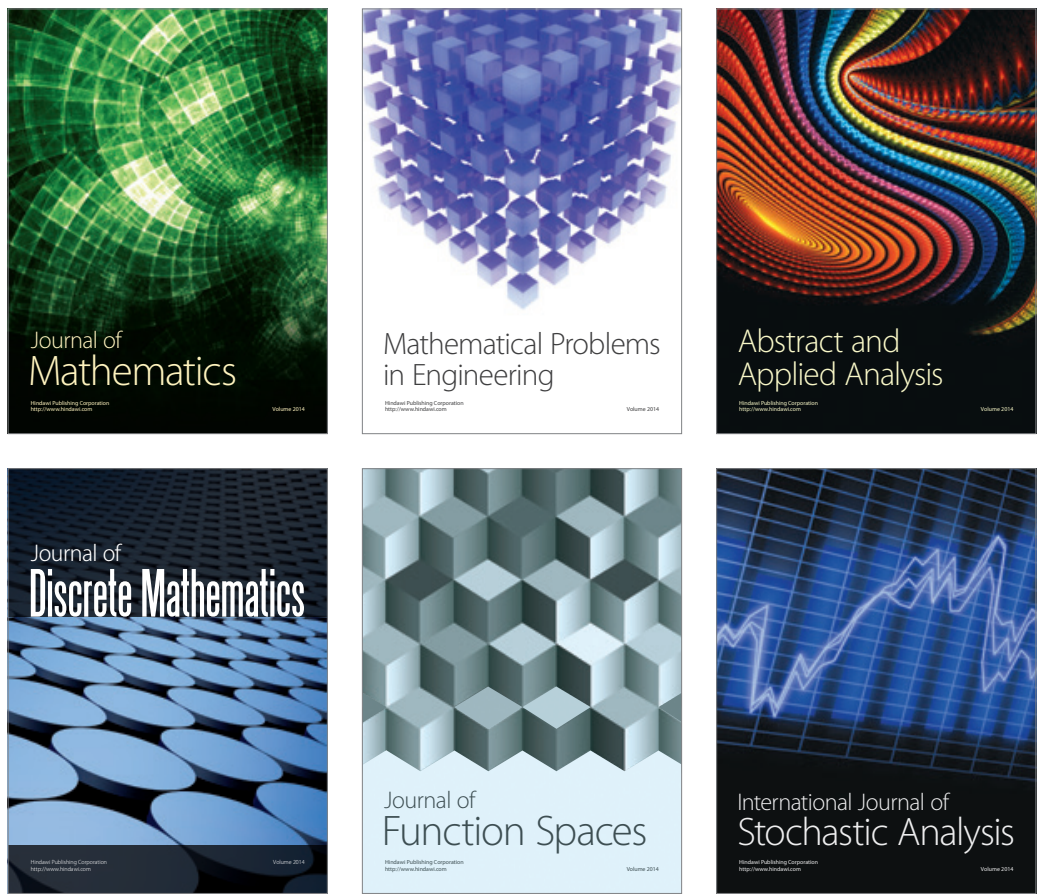

Journal of

Function Spaces

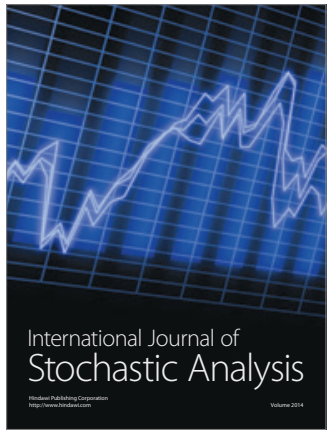

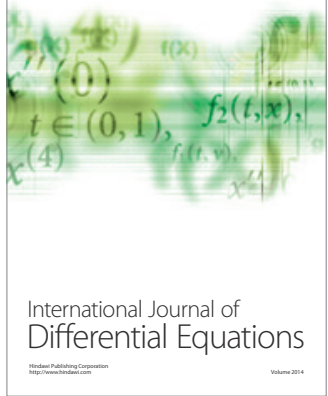
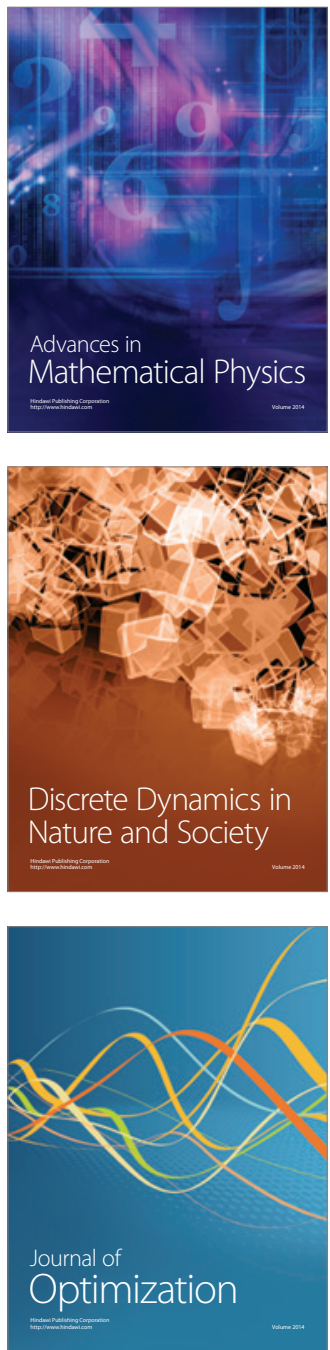